\title{
A New Neutrino Cross Section Data Resource
}

\author{
M.R. Whalley ${ }^{\mathrm{a} *}$ \\ ${ }^{a}$ HEPDATA Database Group and Institute for Particle Physics Phenomenology, \\ Physics Department, University of Durham, Durham City, DH1 3LE, UK
}

\begin{abstract}
We describe a new web based data resource being developed to provide access to accurate and validated cross sections of low energy neutrino and antineutrino interactions. The proposed content of this database are outlined which cover total and differential cross from inclusive, quasi-elastic and exclusive pion production processes from charged and neutral current interactions. Efforts to obtain these data, which come mainly from old bubble chamber experiments, are described as well as the implementation of an embryonic web site to make the resource generally accessible.
\end{abstract}

\section{PREAMBLE}

This talk will introduce a new and developing resource, namely a database of cross sections of low energy neutrino interactions. This is being constructed by the author in collaboration with those listed below.

Several earlier talks at this conference have emphasised the importance of a good knowledge of the low energy neutrino cross sections, the vast majority of which come from bubble chamber experiments performed many years ago. In particular they are needed for Monte Carlo (MC) tuning and model development. It is very important when adjusting the parameters of a MC programme to fit new data, that agreement with older data sets is not lost.

In the past comparisons with data have tended to be qualitative rather than rigorously quantitative. There have been many cross section compilation plots at various workshops [1] and there is a danger of data being transfered from plot to plot with the possibility of inaccuracies creeping in and the original sources of the data being lost. In addition since the data has previously been unavailable in a useful central form, these plots

\footnotetext{
*work done in collaboration with:

C Andreopoulos - CCLRC, Didcot, OX110QX UK

H Gallagher - Tufts U. Medford MA 023155 USA

E Hawker - U. of Cincinnati, Cincinnati OH 45221 USA

M Sakuda - KEK Ibaraki-ken 305-0801 Japan

G Zeller - Columbia U. New York NY 10027 USA
}

have often been arrived at independently by each of the various speakers.

Our aim here is to produce the definitive quantitative database of validated low energy neutrino cross sections together with their associated statistical and systematic errors.

\section{THE DURHAM HEPDATA PROJECT}

\subsection{HEPDATA - introduction}

The development of this data resource is linked to the Durham HEP Database project, HEPDATA, with which the author is involved. A very brief description of the HEPDATA project is given here specifically to demonstrate its relevance to the neutrino data resource.

HEPDATA is a PPARC(UK) funded project which has been in existence now for over 25 years. Its three primary aims have remained essentially the same over this period, namely: (a) To compile scattering data from all types of HEP reactions (cross sections, polarisations, etc...)(b) To make the resulting compilations easily available to the whole community, and (c) To engage in a programme of data commentary and evaluation data reviews.

More recently other services such as the hosting of mirrors of the SLAC SPIRES databases and the Berkeley PDG Review of Particle Physics web pages in the UK, have been added to the HEPDATA operation. HEPDATA also provides 
a unique and comprehensive Parton Distribution Code server. These are all accessible from the main HEPDATA home web page ${ }^{2}$.

\subsection{HEPDATA - database coverage}

The scope of the HEPDATA database covers cross sections from all types of particle physics reactions. It is emphasised that it does not contain "particle properties" which fall into the domain of the RPP of the Berkeley PDG. It also not contain raw data such as found on DSTs of experiments. To appear in the database the data are generally in the final published form. Ideally, to be most useful, they should be fully corrected for acceptances and efficiencies and be model independent. The database contains data from around 10000 publications dating from the 1970s to the present day and is regularly updated. The data are obtained from journals and preprints and direct from the experiments especially when data appear only in graphical form in a publication. In the latter case the authors of the paper are contacted to obtain the exact numerical values shown in the plot. It is very important that this is done at the time of the publication as experience has shown how difficult it is to obtain numerical values at a later date. Data are rarely read from plots due to the difficulty in getting accurate representations of the values, in particular the uncertainties. In its initial phase, over twenty years ago, the HEPDATA project mainly concentrated on cross sections above $2 \mathrm{GeV}$, therefore when attempting to retrieve a complete and reliable collection of decades-old low energy cross section data, special care is needed to ensure completeness, and the latter methods need to be used more frequently. Finally, verification of data entered into the database is always sought from the experimenters themselves.

\subsection{HEPDATA - data reviews}

A development of the work of HEPDATA, which has direct relevance to the subject of this talk, namely the creation of a neutrino data resource, is the involvement of the project in the production of Data Reviews. This fulfils the third stated aim of the project above, the programme

$\overline{2}$ http://durpdg.dur.ac.uk/hepdata/ of data commentary and evaluation. To this end, over the past 15 years the project has produced a series of reviews on a variety of topical and timely aspects of HEP data. Many of these have been published in the IoP's Journal of Physics G, and more recently have been made available on-line where they are kept up-to-date. In these reviews all available data on the topic are presented in both numerical and tabular form together with a short introduction usually written by an expert in the field. There are also comparisons between data sets and, where applicable, comparisons with the relevant theory curves. Topics covered in Data Reviews to date include:

Structure Functions in DIS 2

Drell Yan Cross Sections 3]

EE Correlations in $e^{+} e^{-}$Interactions [4]

Total Cross Sections in $e^{+} e^{-}$Interactions [5] Particle Production in $2 \gamma$ Interactions [6] Hadron Production in $e^{+} e^{-}$Interactions [7] Single $\gamma$ Production in Hadron Interactions 8

Fuelled in part by recent interest shown at the NuInt series of workshops, the Low Energy Neutrino Data resource is envisaged to become the latest in the review series.

\section{THE NEW NEUTRINO DATABASE}

\subsection{Proposed Content}

We have identified the following conditions and limits for data to be included in the database:

- Charged Current (CC) and Neutral Current (NC) data from both $\nu$ and $\bar{\nu}$ interactions.

- $\mathrm{E}_{\nu} \leq 30 \mathrm{GeV}$.

- Total and Differential cross sections from:

- Inclusive reactions $\left(\nu_{\mu} N \rightarrow \mu^{-} X\right.$, etc...).

- Quasi-Elastic (QE) reactions $\left(\nu_{\mu} n \rightarrow \mu^{-} p\right.$, etc...).

- Single $\pi$ exclusive reactions (both incoherent and coherent).

- Two $\pi$ exclusive reactions.

- Fluxes (absolute normalisation and shape.)

Knowledge of the intensity and shape of the incident (anti)neutrino flux and their uncertainties is very important in producing an accurate and reliable database of cross sections. It is particularly 
Table 1

Summary of data from the different experiments

\begin{tabular}{llcc}
\hline Accelerator & Facility & Incl. & QE \\
\hline CERN-PS & Gargamelle & 91011 & 131415 \\
CERN-SPS & Gargamelle & 12 & - \\
& BEBC & 19202122 & {$[23$} \\
& CHARM & {$[2829$} & - \\
& CDHS & {$[32$} & - \\
FNAL & 15ft BC & 333435 & {$[36$} \\
& CCFRR & {$[40$} & - \\
& CCFR & 4142 & - \\
ANL & 12ft BC & 4344 & 4546 \\
BNL & $7 f t$ BC & 505152 & 5153 \\
Serpukhov & SKAT & {$[55$} & {$[56$} \\
& ITEP & 6061 & 626364 \\
& JINR & 65 & - \\
LANL & LSND & 66 & - \\
\hline
\end{tabular}

important for interpreting and using differential cross section distributions from the experiments (which are included in the database). Significant effort will therefore be made to ascertain these for the individual data sets.

\subsection{Locating the data}

In Table 1] we list the accelerators and experiments/facilities where these measurements have been made, indicating the type of data available together with the relevant publication references. The data sources range from the very early Gargamelle experiments at CERN in the 1970's to the more recent experiments at CERN, FNAL and Serpukhov. There is also a very recent inclusive cross section measurement from LSND at LANL.

The relevant data has been collected from a variety of sources. Whenever possible we have obtained the numbers from tables or text in the publications, or used data which was originally supplied by the authors of the papers. This ensures that we have exact and accurate data, including their uncertainties. We have also obtained numbers from private data collections. As a last resort we have read data from plots in the papers. The accuracy and validity of the latter sources of data points is obviously more uncertain and it is our intention to have these verified by physicists who were members of the original experimental collaborations.

\subsection{The MySQL database}

A simple relational database using the MySQL Excldatas)ase management system has been con$161+148$ ed to store the information and data values. The database has only two "database ta23242,2627 The first contains information relevant 3031 he whole paper including the target material, beam energy range and the source of the 373839 as well as more general bibliographic information. The second table contains the actual data points and a field for general comments. 444748 4 4 bles are linked using the IRN number from 57] SLAC/SPIRES database entry for the paper. $5 7 \longdiv { 5 8 9 }$ is a unique and permanent number assigned to every paper by the SLAC library group who manage the SPIRES databases. It is a very useful reference number used also in the HEPDATA databases.

\subsection{The Neutrino Web Site}

From the neutrino database a prototype web site is automatically generated giving user access to the data and information. Figure 1 shows the main home page of this site. The page is basically divided into two main frames plus a navigation frame at the side on the left. This navigation frame, as its name suggests, remains available throughout all other pages of the web site.

From the upper part of this navigation space the user can select data from specific, or all (the initial default), experiments with the relevant information appearing in the upper right hand frame, the Summary Window. Each row in this table represents a publication by the selected experiment with columns for the first author on the paper, the publication reference, the target material and the type of measurement (inclusive, QE or pion(s)). A final column gives links to the relevant plots from the paper.

The publication reference provides a link to the SLAC/SPIRES database record using the IRN number. This gives the user quick access in most cases to the paper itself and other bibliographic information. The columns for the types of measurements contain the neutrino beam energy ranges which are themselves selectable links to the actual numerical data. Selecting one of these links will cause the relevant data to be dis- 


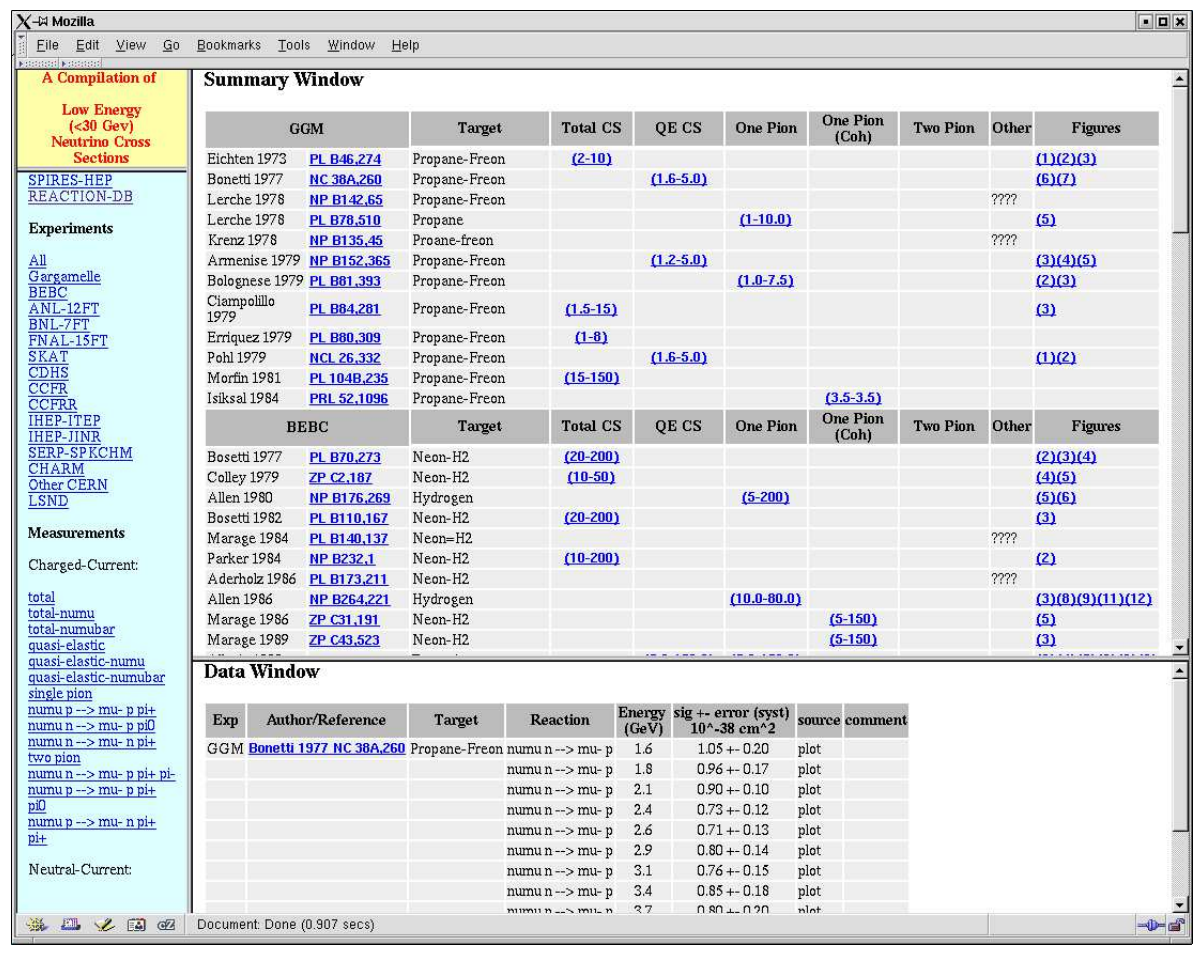

Figure 1. The main home web page of the Neutrino Data Resource ( http://durpdg.dur.ac.uk/hepdata/online/neutrino/)

played in the lower right hand frame, the Data Window. As an illustration, in Figure 1 the result of selecting the QE Gargamelle data from the Bonetti 1977 paper is shown in the Data Window. This appears as a table with a row for each measured data point. The table columns for the experiment name, the author/reference (again linked to the SLAC/SPIRES database), the target material, the reaction, the beam energy, the measured cross section, the source and optional comments. These columns are somewhat self explanatory. The energy is given as the mean value and where available the range is also shown. The cross section values are shown with separate systematic errors where they are given in the papers. The source column indicates how the data was obtained: read from a plot, from numbers in the paper or directly from the authors. As we are still in the process of fully vetting the data, the method of their aquisition should serve as an indicator of the robustness of the numerical values.
The lower half of the navigation space has links to the data displayed in measurement types such as total inclusive, quasi-elastic and single and double pion exclusive, cross sections. Figure 2 shows the result of selecting the QE cross sections. The display changes to a single panel but the columns are similar to those of the Data Window display from the experiments selection. In addition there is the facility on this page to make "on-the-fly" plots of one or more data sets. To do this the user simply ticks the relevant boxes in the "plot" column and then selects any of the plot buttons. At the top the facility to choose the range and type of the scales of the axes of the plots. Figure 3 shows and example of a plot of the total CC inclusive cross sections generated from the database in this way.

\section{Summary}

A new collaborative project has been described to produce a definitive quantitative database of 


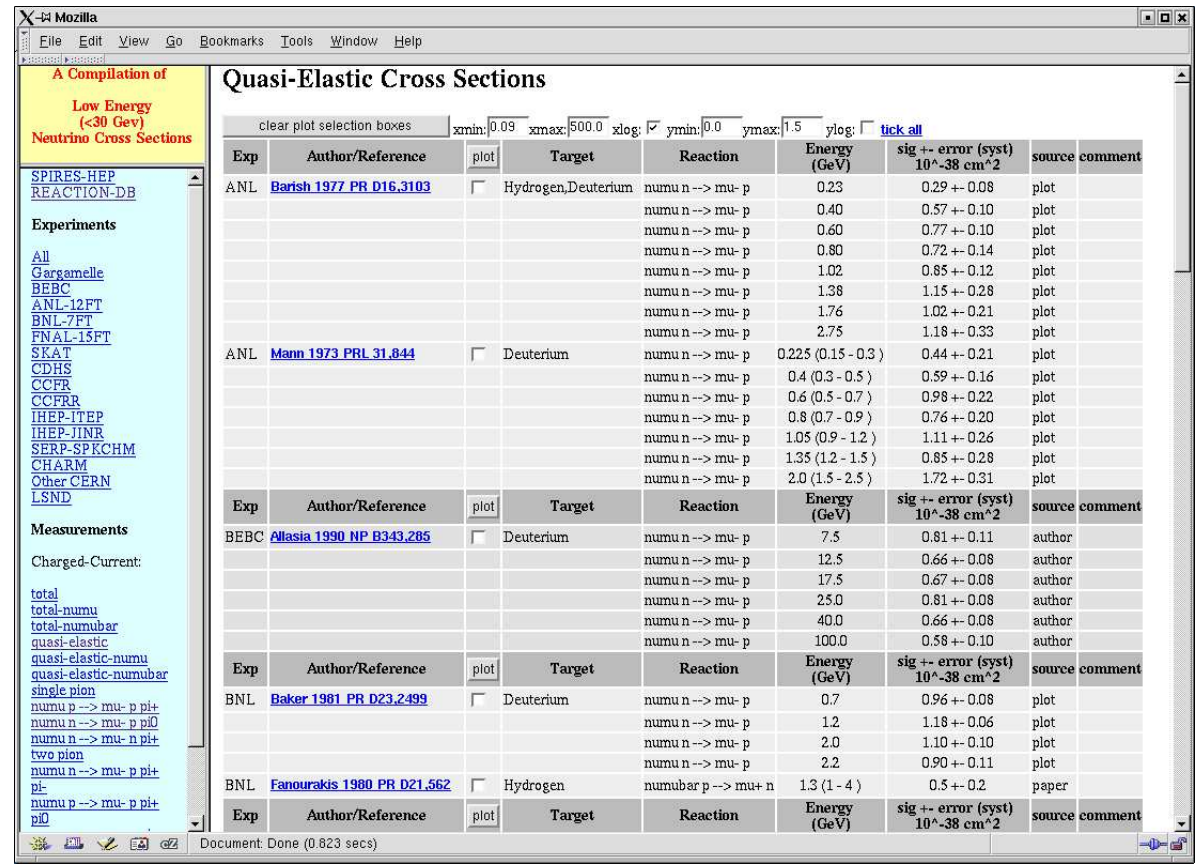

Figure 2. The Quasi-Elastic web page of the Neutrino Data Resource

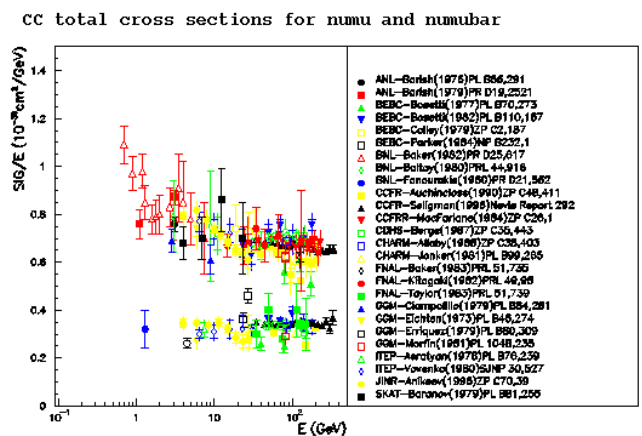

Figure 3. Plot of all CC total inclusive data generated from the database

low energy neutrino cross sections, initially covering total inclusive, QE and single and double pion exclusive, processes. It is the intention to develop the project to include the results of studies of the systematic uncertainties and flux normalisations reported in the papers and in private communications. In achieving the latter we intend to enlist the help of members of the community who worked on the early neutrino experiments and who are still active in the field to provide the best possible verified data.

An embryonic database and web page have been constructed to provide the user with advance and preliminary access to the database. Other methods of accessing and using the data, such as directly from the database, will be developed in the future. One immediate user of the new database will be the Neutrino Monte Carlo Validation Tool, being developed by Costas Andreopoulos, where the output from various MC programs can be "tuned" against existing data. This will be described in the next talk 67.

\section{Acknowledgements}

The author wishes to than all the members of this collaborative effort for their input and help. He also wishes to acknowledge the PPARC(UK) for their continued support of the HEPDATA project under grant number PPA/G/O/2001/00624. 


\section{REFERENCES}

1. M. Sakuda Nucl.Phys.B (Proc.Suppl) 112 (2002)109;

G.P.Zeller NuInt02 - hep-ex/0312061

2. R.G. Roberts and M.R. Whalley J.Phys.G:Nucl.Phys.17(1991)D1

3. W.J. Stirling and M.R.Whalley J.Phys.G:Nucl.Phys.19(1993)D1

4. W.J. Stirling and M.R.Whalley preprint RAL-87-107 (1987)

5. M.R.Whalley J.Phys.G:Nucl.Phys.29(2003)A1

6. M.R.Whalley J.Phys.G:Nucl.Phys.27(2001)A1

7. G.D.Lafferty al.J.Phys.G:Nucl.Phys.21(1995)A1

8. P.Aurenche and M.R.Whalley preprint RAL89-106 (1989)

Gargamelle Data

9. T. Eichten et al. Phys.Lett.B46(1973)274

10. S. Ciampolillo et al. Phys.Lett.B84(1979)281

11. O. Erriquez et al. Phys.Lett.B80(1979)309

12. J. Morfin et al. Phys.Lett.B104(1981)235

13. S. Bonetti et al. Nuovo Cim.A38(1977)260

14. N. Armemise et al. Nucl.Phys.B152(1979)365

15. M. Pohl et al. Lett.Nuovo Cim.26(1979)332

16. W. Lerche et al. Phys.Lett.B78(1978)510

17. T. Bolognese et al. Phys.Lett.B81(1979)393

18. E. Isiksal et al. Phys.Rev.Lett.52(1984)1096 BEBC Data

19. P. Bosetti et al. Phys.Lett.B70(1977)273

20. D. Colley et al. Zeit.Phys.C2(1979)187

21. P. Bosetti et al. Phys.Lett.B110(1982)167

22. M. Parker et al. Nucl.Phys.B232(1984)1

23. D. Allasia et al. Nucl.Phys.B343(1990)285

24. P. Allen et al. Nucl.Phys.B176(1980)269

25. P. Allen et al. Nucl.Phys.B264(1986)221

26. P. Marage et al. Zeit.Phys.C31(1986)191

27. P. Marage et al. Zeit.Phys.C43(1989)523

\section{CHARM Data}

28. M. Jonker et al. Phys.Lett.B99(1981)265

29. J. Allaby et al. Zeit.Phys.C38(1988)403

30. F. Bergsma et al. Phys.Lett.B157(1985)469

31. P. Vilain et al. Phys.Lett.B313(1993)267 CDHS Data

32. J. Berge et al. Zeit.Phys.C35(1987)443 FNAL Data

33. T. Kitagaki et al. Phys.Rev.Lett.49(1982)98

34. N. Baker et al. Phys.Rev.Lett.51(1983)735
35. G. Taylor et al. Phys.Rev.Lett.51(1983)739

36. T. Kitagaki et al. Phys.Rev.D28(1983)436

37. J.Bell et al. Phys.Rev.Lett.41(1978)1008

38. M. Aderholz et al.

Phys.Rev.Lett.63(1989)2349

39. S. Willocq et al. Phys.Rev.D47(1993)2661 CCFRR Data

40. D. MacFarlane et al. Zeit.Phys.C26(1984)1 CCFR Data

41. P. Auchincloss et al. Zeit.Phys.C48(1990)411

42. W. Seligman Thesis NEVIS-292 (1997)

\section{ANL Data}

43. S. Barish et al. Phys.Lett.B66(1977)291

44. S. Barish et al. Phys.Rev.D19(1979)2521

45. W. Mann et al. Phys.Rev.Lett.31(1973)844

46. S. Barish et al. Phys.Rev.D16(1977)3103

47. J. Campbell et al. Phys.Rev.Lett.30(1973)335

48. G. Radecky et al. Phys.Rev.D25(1982)1161; errata ibid D26(1982)3297

49. D. Day et al. Phys.Rev.D28(1983)2714

\section{BNL Data}

50. C. Baltay et al. Phys.Rev.Lett.44(1980)916

51. G. Fanourakis et al. Phys.Rev.D21(1980)562

52. N. Baker et al. Phys.Rev.D25(1982)617

53. N. Baker et al. Phys.Rev.D23(1981)2499

54. T. Kitagaki et al. Phys.Rev.D34(1986)2544 SKAT Data

55. D. Baranov et al. Phys.Lett.B81(1979)255

56. J. Brunner et al. Zeit.Phys.C45(1990)551

57. V. Ammosov et al. Sov.J.Nucl.Phys.50(1989)67

58. H. Grabosch et al. Zeit.Phys.C41(1989)527

59. H. Grabosch et al. Zeit.Phys.C31(1986)203 ITEP Data

60. A. Asratian et al. Phys.Lett.B76(1978)239

61. A. Vovenko et al. Sov.J.Nucl.Phys.30(1979)528

62. S. Belikov et al. Yad.Fiz.35(1982)59

63. S. Belikov et al. Zeit.Phys.A320(1985)625

64. S. Belikov et al. Yad.Fiz.41(1985)919 JINR Data

65. V. Anikeev et al. Zeit.Phys.C70(1996)39 LSND Data

66. L. Auerbach et al. Phys.Rev.C66(2002)015501

67. C. Andreopoulos 'Tools for Neutrino Interaction Model Validation' - NuInt04 - Gran Sasso, March 2004. 